\title{
LA EXPERIENCIA UNIVERSITARIA DE ESTUDIANTES CON GRATUIDAD EN CONTEXTO DE PANDEMIA COVID-19 1
}

\author{
CAROLINA ÁlVAREZ VALDÉS ${ }^{2}$ \\ LEANDRO SEPÚLVEDA VALENZUELA ${ }^{3}$
}

\begin{abstract}
RESUMEN
Este trabajo se centra en la experiencia universitaria de trece estudiantes beneficiarios de gratuidad en universidades altamente selectivas durante el primer semestre online del año 2020, en el marco de las medidas tomadas en el contexto de pandemia del Covid-19. Estos estudiantes habían sido previamente entrevistados, entre los años 2018 y 2019, lo que permite tener una perspectiva comparada. Los hallazgos muestran que la experiencia académica ha sido aún más compleja respecto a su situación previa, pues la modalidad a distancia ha implicado un proceso de adaptación con recursos limitados y una mayor dedicación a sus actividades académicas. La relación con sus pares ha sido reducida, profundizándose la necesidad de la gestión individual de la formación universitaria. En este marco, se evidencia que los soportes institucionales de apoyo a estos estudiantes presentan dificultades de readecuación a las condiciones de la nueva coyuntura, profundizando el efecto de individuación en la experiencia universitaria lejos de la universidad. Finalmente, se identifican los nudos problemáticos más relevantes de las trayectorias socioeducativas de estos/as jóvenes, y sobre esa base, se sugieren iniciativas de políticas complementarias para un mayor impacto de las mismas en el contexto del nuevo escenario socioeducativo que genera la emergencia sanitaria.
\end{abstract}

PALABRAS CLAVES: ESTUDIANTES, EDUCACIÓN SUPERIOR, GRATUIDAD, COVID-19.

${ }^{1}$ Este trabajo fue financiado por la Agencia Nacional de Investigación y Desarrollo (ANID). Programa de Becas Doctorado Nacional/2017-21171452.

${ }^{2}$ Doctora $\bigcirc$ en Educación, Universidad Alberto Hurtado-Universidad Diego Portales. Correo electrónico: caroalvarezvaldes@gmail.com. ORCID ID: https://orcid.org/0000-0003-0402-5072

3 Académico Facultad de Educación, Universidad Alberto Hurtado. ORCID ID: https://orcid.org/0000-0001-9173-7275.Correo electrónico: lesepulv@uahurtado.cl 


\title{
A EXPERIÊNCIA UNIVERSITÁRIA DE ESTUDANTES COM GRATUIDADE NO CONTEXTO DA PANDEMIA COVID-19
}

\begin{abstract}
RESUMO
Este trabalho está centrado na experiência universitária de treze estudantes beneficiados com gratuidade em universidades altamente seletivas durante o primeiro semestre online do ano 2020, no contexto das medidas tomadas no cenário da pandemia COVID-19. Estes estudantes foram entrevistados anteriormente, entre os anos 2018 e 2019, o que permite ter uma perspectiva comparada. As descobertas mostram que a experiência acadêmica tem sido ainda mais complexa em relação à situação anterior, já que a modalidade a distância tem implicado um processo de adaptação com recursos limitados e uma maior dedicação às atividades acadêmicas. A relação com seus pares foi reduzida, aprofundando a necessidade da gestão individual na formação universitária. Neste contexto, é evidenciado que os apoios institucionais a estes estudantes apresentam dificuldades de readequação às condições da nova conjuntura, aprofundando o efeito da individuação na experiência universitária longe da universidade. Finalmente, são identificados os nós problemáticos mais relevantes das trajetórias socioeducativas destes e destas jovens, e sobre essa base, são sugeridas iniciativas de políticas complementares para um maior impacto das mesmas no novo cenário socioeducativo que gera a emergência sanitária.
\end{abstract}

PALAVRAS-CHAVE: ESTUDANTES, ENSINO SUPERIOR, GRATUIDADE, COVID-19

\section{THE UNIVERSITY EXPERIENCE OF STUDENTS WITH FREE TUITION IN THE CONTEXT OF COVID-19 PANDEMIC}

\begin{abstract}
This paper is centered on the university experience of thirteen students who are beneficiaries of free tuition in highly selective universities during the first online semester of 2020, within the framework of the actions taken in the context of COVID-19 pandemic. These students had been previously interviewed, between 2018 and 2019, which allows establishing a comparative perspective. The findings show that the academic experience has been even more complex in comparison to their previous situation, since distance learning modality has implied a process of adaptation with limited resources, a greater dedication to their academic activities and a limited peer relationship that deepens the need of individual management of university learning. In this context, it is evident that the institutional supports for these students are struggling in their processes of readaptation to these new conditions, making the effect of individuation even deeper during their university experience out-of-the-classroom. Finally, the most prominent problematic nodes of the socio-educational trajectories of these male and female young people are identified; from here, we suggest initiatives of complementary policies with a larger impact on the context of the new socio-educational setting generated by the sanitary emergency.
\end{abstract}

KEYWORDS: STUDENTS, HIGHER EDUCATION, FREE TUITION EDUCATION, COVID-19. 


\section{INTRODUCCIÓN}

La pandemia del virus SARS COV 2 (Covid-19) no solo ha provocado graves problemas de tipo sanitarios, exigiendo esfuerzos denodados por el cuidado de la salud colectiva. Junto a esto, su expansión a nivel mundial ha tenido como consecuencia una disrupción en la cotidianidad pocas veces vista en la historia moderna. La necesidad de aislamiento y disminución del contacto entre las personas ha obligado a tomar medidas restrictivas en diversos ámbitos de la vida social. El campo educativo no ha estado ajeno a esto, verificándose por un período prolongado de tiempo el cierre de todo tipo de establecimientos y la implementación de acciones de educación a distancia, no siempre adecuadas y que enfrentan condiciones muy disímiles para su aplicación. La implementación acelerada de nuevas estrategias de educación remota ha estado acompañada de nuevas preguntas investigativas sobre el efecto de estas innovaciones, no solo en el corto plazo, sino que también respecto de sus proyecciones hacia el futuro (Di Pietro et al., 2020).

En efecto, en marzo del año 2020 la Organización Mundial de la Salud (OMS) declaró el Covid-19 como pandemia (IESALC UNESCO, 2020); el mundo globalizado en el que habitamos tuvo que ponerse en pausa. Concretamente en el caso de Chile, a mediados del mismo mes se suspendieron las clases presenciales para el conjunto del sistema preescolar y escolar, extendiéndose posteriormente a las universidades e instituciones de educación técnica superior. Las actividades de docencia y la vida universitaria en su totalidad se trasladaron al hogar y al contexto online, lo que ha implicado una serie de desafíos y también, como se ha afirmado desde los principales organismos internacionales, de oportunidades de innovación en los procesos de enseñanza-aprendizaje tradicionales (IESALC UNESCO, 2020).

Desde entonces, la pandemia y sus efectos en distintos ámbitos de la vida colectiva han sido discutidos en numerosos seminarios, webinars, conferencias y 
publicaciones académicas y no académicas. Tal como afirman Dussel et al. (2020), este tiempo y contexto nos plantean, bajo otro prisma, preguntas simples pero profundas, como, por ejemplo, el efecto de esta situación en la reproducción de las desigualdades y la emergencia de nuevas formas en que estas se pueden evidenciar. Por cierto, el acceso tecnológico a una nueva forma de actividad académica es la más evidente de todas, pero junto con lo anterior, parece necesario profundizar en ámbitos más complejos y que tienen que ver con la experiencia de los actores educativos en todas sus dimensiones.

Bajo este predicamento, este trabajo intenta ser un aporte a la reflexión que el contexto de la pandemia ha generado, dando cuenta de continuidades y cambios en las desigualdades experimentadas por estudiantes de bajos ingresos que recientemente se han incorporado a universidades selectivas en Chile. Es una mirada a la experiencia universitaria en contexto de pandemia, pero también en relación con la experiencia prepandemia de estos jóvenes estudiantes, intentando analizar la incidencia específica de las nuevas condiciones de contexto a un proceso de inserción de por sí complejo.

En concreto, nos centramos en la experiencia universitaria de trece estudiantes que son beneficiarios del sistema de gratuidad $^{4}$ en universidades selectivas de nuestro país, durante el primer semestre online del año $2020^{5}$. Estos estudiantes habían sido previamente entrevistados en dos ocasiones, entre los años 2018 y 2019, en el marco de una investigación de estudios de doctorado destinada a analizar el proceso de integración y permanencia de estos en un

${ }^{4}$ La política de gratuidad comienza a implementarse el año 2016 en el sistema universitario, favoreciendo al $60 \%$ del estudiantado con menores recursos que se inscriba en alguna de las instituciones adscritas a la gratuidad. La gratuidad cubre la duración nominal de la carrera. En caso de requerir un año extra para la finalización de estudios, las instituciones cobran el 50\% del arancel regulado a los/as estudiantes y por más años, el 100\% (Ley Educación Superior, 2018).

${ }^{5}$ En sentido estricto, las entrevistas fueron realizadas al finalizar el primer semestre del año 2020. 
espacio universitario de tipo selectivo. Por tanto, este trabajo ofrece, a partir de entrevistas biográficas y semiestructuradas, una perspectiva comparada de la experiencia universitaria de estos estudiantes, intentado responder a algunas preguntas centrales que permitan abordar la incidencia de las nuevas condiciones de vida impuestas por la emergencia del Covid-19: ¿qué ha cambiado y qué se mantiene en su experiencia estudiantil durante este proceso? ¿De qué manera las disposiciones previas y adquiridas en la experiencia universitaria les han permitido (o no) enfrentar este contexto no previsto? ¿Cuáles son sus condiciones emocionales y materiales para hacer frente a este escenario? ¿Cómo inciden las condiciones actuales en su situación de estudiantes beneficiarios de la política de gratuidad? ¿De qué modo comprenden su experiencia universitaria actual? Sin obviar la especificidad del marco de estudio que da origen a esta secuencia de entrevistas, en este artículo intentaremos abordar el efecto particular de una contingencia inesperada como lo es la pandemia del Covid-19 en la experiencia de vida universitaria de estos jóvenes. Reflexionar sobre la incidencia de lo inesperado en los diversos ámbitos de nuestro interés investigativo constituye, desde este punto de vista, una tarea imprescindible a la luz de los desafíos del tiempo actual. Las interrogantes respecto al proceso de integración de jóvenes de menor nivel socioeconómico en la educación superior deben ser leídas, también, en el marco de esta nueva contingencia. 


\section{HACIENDO FRENTE A LO INESPERADO: ¿QUÉ SABEMOS}

DE LA EDUCACIÓN SUPERIOR EN CONTEXTO ONLINE?

A pesar del carácter emergente del proceso de reorganización de la actividad educativa a un contexto online ${ }^{6}$, a menos de un año de este drástico cambio, es posible identificar investigaciones y reflexiones de académicos/as en el área que remiten a este nuevo escenario y que nos entregan algunas ideas sugerentes para pensar la educación en sus diferentes expresiones. Trabajos recientes realizados en países desarrollados dan cuenta de la preocupación por los efectos en el aprendizaje escolar de las restricciones de acceso al aula, advirtiendo un impacto diferenciado para estudiantes de familias de distintas clases sociales. La presencialidad, desde este punto de vista, aunque no resuelve los problemas de desigualdad, operaría como un factor atenuante de la misma, dadas las condiciones materiales de vida de las familias (Pensiero, Kelly y Bokhove, 2020). De igual manera, otro factor que ha merecido la atención de estudios realizados en el contexto de la pandemia, remite a la capacidad institucional para apoyar el trabajo docente bajo estas nuevas condiciones del quehacer pedagógico; la existencia y disposición de recursos tecnológicos y la generación de un apoyo oportuno y nuevas formas de organización de la actividad profesional, son vistos como variables que han impactado en diferentes formas de adaptabilidad frente a las restricciones existentes (Andrew et al., 2020). Aunque también es posible encontrar indagaciones que, con cierta celeridad, buscan servir de soporte a políticas educativas en este tiempo (véase, por ejemplo, Centro de Estudios MINEDUC, 2021), parece recomendable mantener una actitud crítica y cautelosa a la vez sobre tales efectos y su

\footnotetext{
${ }^{6}$ Por cierto, no se puede obviar el hecho de que desde hace décadas existe una oferta amplia de formación a distancia en el desarrollo de diversos programas curriculares de educación superior. Con todo, la necesidad de readecuación urgente de la oferta formativa tradicional de los centros educativos a diversas alternativas de tipo remota, constituye un hecho inédito y particular de este período provocado por la emergencia sanitaria. Este es el campo y el contexto de preocupación de este trabajo.
} 
incidencia en el tiempo, sobre todo, porque la propia expansión temporal de la pandemia (por lo menos al momento de escribir este trabajo) es aún de pronóstico incierto.

Ahora bien, los estudios empíricos sobre el contexto online en la educación superior dan cuenta de varias problemáticas concretas. Primero, la falta de dispositivos tecnológicos y deficiente conectividad en la mayoría de los países de América Latina (Cannellotto, 2020; IESALC UNESCO, 2020; Ordorika, 2020), así también como de diferencias importantes al interior de los países, viéndose afectadas principalmente las zonas rurales y aisladas y los sectores más pobres de los centros urbanos (Aristovnik et al., 2020). Por ello, existe preocupación en los organismos internacionales respecto al aumento de la brecha social, ya existente en materia de educación, que esta crisis estaría agudizando (IESALC UNESCO, 2020; Pedró, 2020). Segundo, la emergencia y la urgencia en el paso a la educación online como manera de dar continuidad a los estudios en el nivel de educación superior no ha implicado, necesariamente, una adecuación pedagógica, sino que ha sido, más bien, una extensión del aula presencial. Incluso se ha acuñado el anglicismo coronateaching para dar cuenta de este fenómeno (IESALC UNESCO, 2020; Pedró, 2020; Pérez Centeno, 2020). En la literatura reciente, también se destaca la fuerte autodisciplina que exige a los estudiantes esta modalidad online de estudios (Hussein et al., 2020, Pedró, 2020). Este hecho constituye una cuestión aún más compleja para estudiantes que deben hacerse cargo de cuidados de hijos/as y de personas mayores y/o enfermas (Mishra et al., 2020; Ordorika, 2020) y estudiantes que son el sustento de sus hogares y/o de sí mismos, los que deben buscar la forma de continuar trabajando y generar ingresos en el marco de la crisis económica derivada de la emergencia del Covid-19 (Cannellotto, 2020).

Junto con ello, estas indagaciones dan cuenta de los fuertes impactos emocionales de la pandemia en estudiantes y docentes; esto se evidencia en la 
sensación de agobio por las nuevas tareas y el deber de usar distintas plataformas y medios digitales a los que muchos no tenían cercanía o entrenamiento suficiente (IESALC UNESCO, 2020; Pedró, 2020), e incluye sensaciones de angustia y rabia debido a la presión cotidiana y adaptación a una nueva realidad (Aristovnik et al., 2020). A todo esto se suma el miedo al futuro profesional, pues la salida al mundo laboral se ve compleja para estudiantes egresados en una sociedad en crisis, luego de esta pausa en las actividades económicas (OECD, 2020).

Estos trabajos encienden las alertas sobre varios puntos a tener en consideración para la educación superior (en adelante, ES) en este nuevo contexto y dan cuenta de variadas dimensiones sobre las que es necesario analizar los desafíos de la formación universitaria en el futuro inmediato (Aristovnik et al., 2020). Sin embargo, estas indagaciones emergentes entregan menos pistas para pensar las experiencias estudiantiles como parte de un entramado social más complejo y local, existiendo vacíos de conocimiento acerca de qué modo se ha ido acomodando la vida cotidiana de las nuevas generaciones de estudiantes en este nuevo contexto. Si bien las nuevas condiciones de la vida estudiantil generadas por la pandemia son un fenómeno global, ya sabemos que tienen expresiones diversificadas; dar cuenta de estas experiencias y aventurar un análisis sobre sus proyecciones futuras, se vuelve así, un desafío relevante para ahondar en los sentidos y sinsentidos de esta nueva realidad y su efecto, posiblemente desigual, entre quienes han tenido que enfrentar una nueva normalidad.

La siguiente sección está destinada a discutir la noción sociológica de experiencia para consolidar una perspectiva propia que permita interpretar los relatos de los/as estudiantes que fueron parte de este trabajo. 


\section{LA NOCIÓN DE EXPERIENCIA PARA LA INVESTIGACIÓN EN} EDUCACIÓN SUPERIOR

En este trabajo recurrimos a la noción de experiencia para referirnos al conjunto de actividades, rutinas y significaciones que construyen los/as estudiantes en este contexto de vida universitaria vía remota u online. Aunque la noción de experiencia ha sido abordada desde distintas vertientes teóricas y campos de estudios (Dubet, 2010; McNay, 2004; Scott, 2009), para fines de este trabajo nos enfocamos en aquella que ha sido mayormente desarrollada en investigaciones de las nuevas generaciones de estudiantes en la educación superior.

La noción de experiencia tiene una larga tradición en la filosofía y los estudios sociales. En el último tiempo ha sido retomada por autoras feministas postestructurales (McNay, 2004; Scott, 2009). Dentro de las últimas elaboraciones, encontramos la noción de experiencia desarrollada por McNay (2004), a partir de la consideración del concepto de habitus y la fenomenología del espacio social de Pierre Bourdieu. Desde esta perspectiva se entiende la experiencia como relaciones sociales vividas, para dar cuenta del modo en el que las estructuras se expresan en actos cotidianos y reiterativos. En este marco, la experiencia resulta ser un concepto intermedio que permite comprender la agencia de los sujetos sin desestimar el condicionamiento de las estructuras sociales. Por otra parte, la línea teórica de la sociología de la experiencia, desarrollada por Dubet (2010), considera el modo en el que los sujetos se convierten en actores por medio de la conjugación de distintas lógicas de acción, asociadas a los roles, estrategias y procesos de subjetivación. En este caso, la experiencia universitaria corresponde al modo en el que los estudiantes se van convirtiendo en estudiantes universitarios, enfrentando las diversas pruebas que posibilitan la integración o no a estas instituciones de alto prestigio. Desde esta perspectiva, la construcción de un proyecto vocacional profesional requiere de 
sujetos con mayores niveles de reflexividad para hacer frente al conjunto de retos que enmarcan su quehacer como individuos.

En relación con la reflexividad de los sujetos y el estudio de las experiencias universitarias, los trabajos empíricos de Reay (Reay, 2010; Reay et al., 2009, 2010) sobre estudiantes de clases trabajadoras en universidades de élite retoman los postulados de Bourdieu, pero otorgándole un lugar central a la reflexividad de los agentes, sin reducirla al desajuste habitus-campo social como lo hace Bourdieu-, sino que como parte de las experiencias más cotidianas de los individuos (Archer, 2007; McNay, 1999). Es más, por medio de esta reflexividad pueden afrontar la experiencia universitaria, reconociendo su posición social, su relación con las instituciones y pares, y las transformaciones que desarrollan en su paso por las instituciones de ES.

Esta perspectiva encuentra un punto de intersección con lo planteado por Lahire $(2004,2008)$ acerca de la pluralidad de contextos a los que se enfrentan los/as jóvenes en la sociedad actual, por lo que la disonancia entre prácticas y posiciones sociales sería más común. Por lo mismo, podrían no necesariamente producirse habitus desgarrados en este tránsito por contextos plurales. No obstante, los trabajos que han abordado la experiencia estudiantil en instituciones prestigiosas y de élite siguen reconociendo los desajustes de habitus de los estudiantes cuando estos se enfrentan a campos sociales poco familiares, principalmente considerando la clase social como diferenciador de experiencias.

Si bien esta perspectiva analítica corresponde a estudios y revisiones teóricas de países del norte, recientemente en América Latina y Chile se ha constituido un campo temático sobre la ES y la experiencia de estudiantes, con áreas de estudio clásicas como las referidas al acceso, retención y rendimiento académico, desde una diversidad de referencias teóricas (Guzmán-Valenzuela et al., 2020). En ese marco, en el último tiempo se ha desarrollado una línea investigativa que considera las nociones de habitus, campo social y habitus 
institucional para dar cuenta del complejo tránsito de estudiantes de menor nivel socioeconómico en instituciones de prestigio, que exigen altos niveles de agencia asociada a procesos de reflexividad y sacrificio de su parte (Gallardo et al., 2014; Leyton et al., 2012).

Este trabajo se sitúa en este último grupo, persiguiendo la comprensión de la experiencia en un sentido material, vinculado a las estructuras sociales (campos sociales de origen y de destino), la expresión de los habitus en prácticas cotidianas, y el sentido que le dan a estas prácticas y a la experiencia universitaria en tiempo presente y proyectivamente en términos profesionales. En síntesis, entendemos la noción de experiencia con una pretensión de totalidad, es decir, atendiendo a los ámbitos académicos, sociales, extra-académicos, profesionales y en relación con su origen social.

\section{Metodología}

Este artículo es parte de una investigación más amplia sobre la experiencia de estudiantes con gratuidad en universidades altamente selectivas. Se recurrió al enfoque biográfico para dar cuenta de las experiencias, puntos de quiebre y transformaciones de los/as estudiantes en su relación con las instituciones educativas, familia y pares (Bertaux, 2005; Rojas, 2008; Toledo, 2002). En ese marco, fueron realizadas entrevistas biográficas que permitieron un acercamiento al relato de la persona sobre su propia vida, tal y como la cuenta (Bertaux, 2005; Rojas, 2008). En este enfoque resulta relevante llegar a la producción de «buenas historias», entendidas por Merrill y West (2009) como ricas en detalles y de alta reflexividad.

Los/as estudiantes que fueron parte de la investigación cursan estudios en las dos universidades más selectivas y prestigiosas, ubicadas en el centro del país (Brunner, 2009; Orellana, 2011; Torres y Zenteno, 2011). Pues, a pesar de la 
masificación de la ES en el país, las mejores posiciones continúan estando reservadas para los grupos con mayores capitales, que ingresan a universidades prestigiosas. La literatura internacional identifica la experiencia de los/as estudiantes de sectores de menores recursos y capitales en este tipo de centros educativos como desajustadas, es decir, que sus habitus son menos afines a este campo social en comparación con sus pares de mayor capital, y por lo mismo pueden propiciar mayores niveles de reflexividad y un fuerte trabajo sobre sí mismos (Abrahams y Ingram, 2013; Lehmann, 2009; Reay et al., 2009).

Para llevar a cabo esta investigación fueron entrevistados en distintos momentos de su trayectoria educativa trece estudiantes con gratuidad de las carreras de Derecho, Medicina, Kinesiología y Trabajo Social de estas universidades (ver cuadro 1). Estas carreras se definieron para analizar eventuales diferencias de experiencias entre ellas, unas mayormente asociadas a la tradicional reproducción de élites y otras no (Villalobos et al., 2020), aunque igualmente exigentes respecto a su oferta en otros planteles universitarios. Los/as estudiantes que fueron convocados a ser parte de la indagación debían ser beneficiarios de la política de gratuidad, es decir, fue una convocatoria amplia, con lo cual se buscó posibilitar una diversidad de estudiantes y trayectorias, para analizar sus modos de afrontar las distintas carreras y la vida universitaria. Las entrevistas (particularmente las desarrolladas durante los años 2018 y 2019) indagaron en las experiencias educativas, con especial foco en los ámbitos de financiamiento, desempeño académico, y relaciones sociales con el entorno estudiantil y del cuerpo de docentes. El total de trece entrevistas que se efectuaron al finalizar el primer semestre del año 2020, aunque abordaron estos mismos tópicos, también focalizaron su interés en la situación generada por la pandemia del Covid y el efecto de esta emergencia en la vida universitaria de los estudiantes. Cada entrevista tuvo una duración aproximada de una hora y todas las conversaciones fueron transcritas para su posterior comparación con el material previamente recogido en los años anteriores. 
Los/as participantes de la investigación, tal como se observa en el cuadro 1, corresponden a estudiantes de niveles socioeconómicos medios bajos, de escuelas de distinta dependencia y modalidad. Dentro de este grupo destacan estudiantes de liceos académicamente selectivos, con una fuerte impronta de continuidad de estudios en instituciones superiores selectivas y dos estudiantes de establecimientos particulares pagados (no de élite ${ }^{7}$ ). Finalmente, los/as estudiantes cursan distintos años de la carrera, por lo cual sus años de ingreso son diferentes.

CUADRO 1. CARACTERÍSTICAS ESTUDIANTES ENTREVISTADOS/AS

\begin{tabular}{|c|c|c|c|c|c|}
\hline Nombre & Universidad & Carrera & Año & $\begin{array}{l}\text { Establecimien } \\
\text { to educacional } \\
\text { EM }\end{array}$ & Clase social ${ }^{8}$ \\
\hline Lorena & US1 & Trabajo Social & $4^{\circ}$ & $2 \times 1$ & Media-baja \\
\hline Javier & US1 & Trabajo Social & $3^{\circ}$ & Municipal CH & Media-baja \\
\hline Johanna & US2 & Trabajo Social & $4^{\circ}$ & PS-TP & Media-baja \\
\hline Luis & US2 & Trabajo Social & $2^{o}$ & $\begin{array}{l}\text { Particular } \\
\text { pagado }\end{array}$ & Media-media \\
\hline Andrea & US1 & Derecho & $5^{\circ}$ & PS-TP & Media-baja \\
\hline Roberto & US1 & Derecho & $4^{\circ}$ & $\begin{array}{l}\text { Liceo } \\
\text { Municipal } \mathrm{CH} \\
\text { selectivo }\end{array}$ & Media-baja \\
\hline Pedro & US2 & Derecho & $1^{o}$ & $\begin{array}{l}\text { Liceo } \\
\text { Municipal } \mathrm{CH} \\
\text { selectivo }\end{array}$ & Media-media \\
\hline Marcelo & US2 & Derecho & $1^{\circ}$ & $\begin{array}{l}\text { Liceo } \\
\text { Municipal } \mathrm{CH} \\
\text { selectivo }\end{array}$ & Media-baja \\
\hline Guillermo & US2 & Derecho & $4^{\circ}$ & $\begin{array}{l}\text { Liceo } \\
\text { Municipal CH }\end{array}$ & Media-baja \\
\hline Enrique & US1 & Medicina & $4^{\circ}$ & $\begin{array}{l}\text { Particular } \\
\text { pagado }\end{array}$ & Media-media \\
\hline
\end{tabular}

${ }^{7}$ PNUD (2017) define catorce establecimientos de élite particular pagados.

${ }^{8}$ La clase social se definió a partir del nivel educativo, ocupación y residencia de las familias de los/as estudiantes, variables claves consideradas en las distintas investigaciones sobre estratificación social (Espinoza y Barozet, 2008; Torche y Wormald, 2004). Las clases medias-bajas corresponden a estudiantes cuyas familias nucleares tienen estudios secundarios completos e incompletos, se desempeñan en trabajos de baja cualificación y residen en zonas de niveles socioeconómicos mediosbajos. Las clases medias-medias corresponden a familias en que unos de los padres cuenta con estudios superiores universitarios, por lo que se desempeñan en trabajos de mayor cualificación; no obstante, los lugares de residencia corresponden a sectores de clases medias emergentes, no a sectores de clases medias profesionales (Stillerman, 2016). 


\begin{tabular}{|c|c|c|c|c|c|}
\hline Cristina & US1 & Medicina & $4^{\circ}$ & PS-CH & Media-baja \\
\hline Loreto & US1 & Kinesiología & $1^{\circ}$ & $\begin{array}{l}\text { Liceo } \\
\text { Municipal CH } \\
\text { selectivo }\end{array}$ & Media-baja \\
\hline Bastián & US2 & $\begin{array}{l}\text { Bachillerato/D } \\
\text { erecho/Trabaj } \\
\text { o Social }\end{array}$ & $4^{\circ}$ & PS-CH & Media-baja \\
\hline
\end{tabular}

Nota: Universidad Selectiva 1 (US1); Universidad Selectiva 2 (US2);

Científico-Humanista (CH); Técnico Profesional (TP); Particular

Subvencionado (PS).

Para el procesamiento del material que sirve de base a este artículo, primero fue realizado un análisis temático de las entrevistas, en el que se buscó no perder el sentido de las narraciones realizadas por los y las estudiantes (Merrill y West, 2009; Sautu, 1999). En este ejercicio, los ejes temáticos de la experiencia educativa fueron analizado nuevamente por medio de análisis de contenido, con lo cual se indagó en los contenidos explícitos e implícitos de las entrevistas (Andréu, 2002). Este último análisis fue realizado con el software N.vivo 12.

\section{Resultados}

Los resultados son presentados considerando la experiencia universitaria de los estudiantes en un sentido amplio, con un foco principal en los ámbitos de la vida académica, social y material, además de algunas consideraciones emergentes en relación con el contexto de trabajo online. Como podrá verse, de manera general, los/as estudiantes perciben que la experiencia universitaria, por una parte, ha estado centrada de manera exclusiva en el ámbito académico, cuestión acrecentada por el contexto de pandemia; y por otra parte, que esta vida universitaria académica, pese a verificarse fuera de las aulas tradicionales, ha ocupado gran parte de su tiempo. 


\subsection{LA EXPERIENCIA UNIVERSITARIA PREVIA A LA PANDEMIA}

A partir de las entrevistas realizadas en las etapas previas a la crisis de Covid, es posible indicar algunas características predominantes de las experiencias de los/as estudiantes en estas instituciones altamente selectivas y prestigiosas. En términos de su experiencia académica, en primer lugar, los/as estudiantes dan cuenta de un shock inicial ante la cantidad y complejidad de los contenidos de las clases universitarias. Todos señalan que existe un descenso en sus rendimientos académicos en comparación a la trayectoria escolar previa. Sin embargo, algunos/as logran estabilizarse rápidamente, entre el primer y segundo semestre del primer año o, a más tardar, durante el segundo año. En general, quienes tienen más dificultades en el ámbito académico provienen de establecimientos educacionales con menos foco en lo académico y con posibilidades de continuidad de estudios en instituciones superiores no selectivas (como ocurre entre quienes estudiaron en la modalidad técnico profesional o quienes terminaron la enseñanza media en programas especiales); pero más difícil aún es esta situación para quienes deben combinar estudios y trabajo, y estudios, trabajo y maternidad. Es decir, aunque todos manifiestan que su experiencia estudiantil ha sido particularmente difícil, quienes tienen condiciones materiales más complejas son quienes viven experiencias al filo entre la continuidad y el abandono. Para hacer frente a esta situación y lograr una mejor adaptación académica, los testimonios destacan la importancia de lograr una fuerte disciplina académica, probar y ensayar metodologías de estudios eficaces, así como también, resultan relevantes las tutorías y apoyos institucionales en este proceso de ajuste ${ }^{9}$. El acceso y disponibilidad de las instalaciones e infraestructura de las universidades son mencionados como un factor material

\footnotetext{
${ }^{9}$ Por lo general, se trata de iniciativas de tutorías de estudiantes de nivel superior u otras instancias de apoyo de las propias instituciones para favorecer la retención. Así como programas ministeriales en asociación con instituciones de ES como ocurre con el Programa de Acceso a la educación superior, PACE, del Ministerio de Educación.
} 
clave, lo que les permite desarrollar de mejor manera sus jornadas de estudio en condiciones favorables que no encuentran en el espacio de sus hogares.

Ahora bien, respecto a la experiencia social en este nuevo escenario educativo, la gran mayoría de los entrevistados señala haber tenido problemas para entablar relaciones de amistad en su paso por la universidad, principalmente, debido a la dedicación casi exclusiva a los estudios, quedando otro tipo de actividades relegadas a un segundo plano. Junto con ello, en algunos casos, sobre todo en carreras más altamente selectivas, se producen cierres sociales de parte de los grupos de más altos capitales. No obstante, muchos/as mantienen relaciones de amistad, y particularmente conforman grupos de trabajo y estudio con pares de condiciones socioeconómicas similares, que pasan a ser soportes relevantes para sobrellevar la vida académica de alta exigencia. Las emociones en este paso por la universidad son de agobio, sensación de insuficiencia y soledad. Es un camino que muchos/as viven con gran dificultad, pero que a la vez posibilita la apertura del mundo, hacia otras personas, lugares, pensamientos políticos y sociales, de gran riqueza en su experiencia vital, que posibilitan futuros profesionales antes no imaginados.

En general, ser beneficiarios/as de la política de gratuidad es comprendida como una situación de menor presión financiera familiar, sobre todo considerando el alto costo de endeudamiento que ha caracterizado el sistema de ES en Chile en las últimas décadas (Kremerman y Páez, 2016; Pérez-Roa, 2014). Junto con ello, las condiciones de implementación de la política han implicado para los/as estudiantes una presión por no retrasarse más allá de los años formales de duración ante el riesgo de perder el beneficio de la política pública. Para tal efecto, se señala que es necesario recurrir a distintas estrategias; como, por ejemplo, cursar más cursos por semestre, cuestión que agudiza la focalización personal exclusiva en el ámbito de las actividades estrictamente académicas. Para algunos de los estudiantes que se encuentran en etapas avanzadas de sus carreras, la alternativa de acceder a un crédito financiero para la finalización de sus 
estudios emerge como una posibilidad cierta, toda vez que se trata de un proceso altamente exigente.

En definitiva, encontramos una experiencia universitaria marcada por la dedicación académica y el aprendizaje de convertirse en estudiantes altamente exigidos. En este proceso resultan relevantes algunos mecanismos institucionales de apoyo y, muy particularmente, la disponibilidad de recursos materiales en los centros de estudios que son fundamentales para su quehacer cotidiano. De igual modo, sobre todo en los primeros años, se trata de una experiencia social de baja integración y con un proyecto profesional vocacional, que en muchos casos va construyéndose en el paso por la universidad y sobre todo en los años más avanzados de la carrera.

\subsection{EXPERIENCIA ACADÉMICA EN EL CONTEXTO ONLINE}

\subsubsection{El contexto online: de sincronías y asincronías}

Las palabras sincrónico y asincrónico constituyen parte de un vocabulario emergente en el discurso de los/as jóvenes estudiantes. Dichos conceptos, por cierto, refieren a las dimensiones de tiempo y espacio, todo lo que se vio trastocado con esta nueva experiencia universitaria virtual. En este caso, la sincronía implica estar en el mismo momento en el mismo lugar virtual, mientras que lo asincrónico implica lo contrario, refiere a aquello que ya ocurrió en un tiempo pasado. Las clases asincrónicas remiten a la posibilidad de observar (no de asistir) clases después de que estas hayan sido realizadas, pensadas sobre todo para quienes no cuentan con tiempo ni conexiones a internet estables, es decir, para quienes evidencian las condiciones de mayor precariedad para enfrentar este escenario. Este nuevo contexto, que poco a poco fue desenredándose en diferentes formas de continuidad en las distintas instituciones universitarias, implicó una primera decisión para los/as estudiantes: ¿seguir o no seguir? En el 
caso de los estudiantes entrevistados, la continuidad de sus estudios en el semestre online está condicionado por varias razones: el financiamiento de la ES, «no perder el año» y así poder finalizar a tiempo la carrera y, con ello, salir al mundo laboral lo antes posible.

El tiempo y el espacio para continuar con la vida universitaria ha implicado para los/as estudiantes un gran esfuerzo y altos niveles de agencia y autocontrol. Algunos han podido dedicarse completamente a sus actividades académicas, mientras que otros han debido trabajar y estudiar a la par, y en el caso de una de las estudiantes entrevistadas, trabajar, criar y estudiar. El contraste es importante; el tiempo y el espacio en la vida universitaria presencial les llevaron a conocer en profundidad sus campos profesionales, otros lugares y a otras personas, con lo cual se abrieron nuevos mundos y posibilidades imaginadas. La pandemia los devolvió a sus hogares y con ello al espacio anterior, ¿qué implicancias ha tenido en distintos ámbitos este retorno y llevar la universidad al hogar?

\subsection{2 ¿El aprendizaje en pausa?: la relación con el conocimiento}

Los/as estudiantes dan cuenta de ciertas dificultades y otras ventajas respecto de las clases online en contexto de pandemia. Entre las dificultades principales destaca la calidad del aprendizaje; la gran mayoría señala que más bien se han enfocado en cumplir con las tareas y evaluaciones de los distintos cursos, pero que el aprendizaje ha quedado en un segundo plano. Incluso perciben que los contenidos revisados en las distintas carreras son volátiles («ramo pasado, ramo olvidado». Guillermo, Derecho US2, 2020). Esta merma en la calidad del aprendizaje tiene relación con la modalidad de las clases, las condiciones materiales de estudio, las problemáticas de estar expuestos a condiciones extraordinarias, entre otros asuntos.

En el contexto previo a la pandemia, para sobrellevar de buena manera los distintos cursos de sus carreras, los estudiantes desarrollaron una fuerte 
disciplina, por medio de la incorporación de rutinas de estudio estrictas. En un proceso de ensayo y error fueron descubriendo las mejores formas de estudiar y con el paso de los semestres adquirieron disposiciones al aprendizaje más fuertes. En la adquisición de estas rutinas y estrategias de estudios, resultó clave contar con los espacios y recursos de sus centros universitarios como las bibliotecas, salas de estudio, libros, material impreso, apoyos de tutores pares y sobre todo la posibilidad de conversar con docentes y compañeros. Por todo esto, esos aprendizajes y adquisición/actualización de disposiciones académicas más fuertes se ponen en tensión e incluso en suspenso en este nuevo contexto, tanto para estudiantes que cursan los primeros como los últimos semestres de la carrera.

Encuentro que por lo menos en segundo semestre del primer año, cuando ya había agarrado más el ritmo, ahí sentía que por ejemplo, requería de menos esfuerzo para poder aprenderme la materia, para memorizar un poco más de conceptos, ahora encuentro que tuve que, no sé si partir de cero porque ya tenía la base, como saber cómo estudiar, pero sí tuve que poner un poquito más de mi parte, esforzarme un poquito más, para rendir de la misma manera que lo hacía. (Pedro, Derecho US2, 2020)

Este esfuerzo de continuar el año académico de parte de las instituciones, de igual modo es comprendido por algunos estudiantes como un año perdido en términos de aprendizajes y de conocimientos necesarios para desarrollarse en el ámbito profesional una vez egresados. Particularmente complejo resulta para estudiantes de los últimos años de Trabajo Social, Derecho y Medicina, quienes les dan gran importancia a los espacios de práctica, ya que reconocen que se trata de la posibilidad de aprender a aplicar sus conocimientos teóricos con la guía de docentes y el apoyo de sus pares. La práctica constituye el paso entre la universidad y el mundo laboral, experiencia que se ve compleja y de difícil adaptación bajo la modalidad online. Además, está presente la sensación de que las restricciones al ejercicio de la práctica profesional ya no pueden ser resueltas, 
porque los planteles decidieron continuar con la oferta curricular sin mayores modificaciones, constituyéndose en una pérdida irreparable:

Siento que este año para mí fue perdido en todo sentido, aparte de la práctica, siento que yo lo perdí, como que este año no me va a aportar mucho en nada, porque los aprendizajes que tuve en la $U$, de verdad que no fueron los aprendizajes que yo esperaba. (Johanna, TS US2, 2020)

Entonces, está esta discordancia en que nadie nos va a resguardar y nadie nos va a proteger frente a las embarradas que nos vamos a mandar, cuando seamos internos y no sepamos nada o los internos cuando salgan a trabajar y no sepan nada. Porque nadie te puede justificar después cómo: «¡Ay perdone! Es que yo soy de la generación Covid, no sé evaluar». (Enrique, Medicina US1, 2020).

De acuerdo con los testimonios, los resultados en sus rendimientos académicos han sido disímiles, si bien todos afirman dedicar más horas de estudio para obtener resultados similares a un semestre presencial. Algunos estudiantes han renunciado a cursos o reprobado al final del semestre, mientras que otros han subido sus promedios. En este sentido, los estudiantes de los años más altos de las carreras se sienten con más confianza y ven difícil reprobar en el momento del trayecto en el que se encuentran, pues ya sortearon los años de mayor complejidad. A diferencia de esto, los estudiantes de los primeros años han tenido una experiencia mucho más irregular, con mayor recurrencia de reprobación o descenso de sus notas. Muchos argumentan que en su experiencia universitaria aún no terminaban de ajustarse completamente a las exigencias académicas, cuando debieron adaptarse, además, a estas nuevas formas de vida estudiantil.

De todos modos, no es posible identificar una tendencia unívoca; Andrea (Derecho US1) considera que esta modalidad online le ha permitido tener más tiempo para dedicarse a estudiar, dado que no tiene que movilizarse de su casa a la facultad, cuestión que podría tomarle unas cuantas horas al día. Asimismo, ha mejorado sus habilidades escritas, ya que este contexto ha promovido la 
realización de un número mayor de trabajos de este tipo. Mientras que en el caso de Cristina (Medicina US1), si bien también señala tener más tiempo ya que no debe movilizarse diariamente, de algún modo percibe tener menos tiempo y aprender mucho menos; para ella todos esos espacios y tiempos entre clases presenciales y después de estas constituían momentos relevantes para su bienestar y aprendizaje. Todo ello se perdió en este contexto online, de mayor soledad e individuación.

Particularmente, en este semestre online la gestión del tiempo en el hogar ha sido un tema complejo; las distracciones que existen en estos espacios, además de las preocupaciones domésticas, dificultan la concentración y el estudio. Finalmente, un asunto relevante que ha generado complicaciones en el aprendizaje de los/as estudiantes tiene relación con problemas de malestar psicológico que han enfrentado en esta pandemia. A estos elementos, se suman las preocupaciones económicas, el miedo a la pérdida de empleo de los sostenedores de familia o congeniar estudios y trabajo, dado que muchos deben o han debido ayudar con la economía de sus hogares. Ambas problemáticas son también identificadas por las indagaciones en contexto Covid-19 (Aristovnik et al., 2020; Cannellotto, 2020; Mishra et al., 2020; Ordorika, 2020).

En este marco, para varios entrevistados, el estudio, la carrera y el estar todo el día frente a una pantalla perderían sentido, más aún en el marco de una propuesta pedagógica desde sus propios centros de estudios, que rápidamente adoptaron una nueva normalidad en la forma de educación remota. Como lo indica Enrique en la cita siguiente, el efecto de este proceso genera sensaciones de perplejidad; las respuestas adaptativas son abordadas individualmente y en el seno del hogar - es decir, en ausencia de conversaciones mayores - sin que exista, siquiera, un contraste de experiencias con los propios compañeros de estudio, sujetos cercanos, pero distantes en la experiencia educativa bajo la pandemia. 
Se siente plástico estudiar, se siente muy fútil eso también, lo mismo que tenía que ver con la falta de práctica, como que se siente muchas veces desmotivante, porque para qué me estoy leyendo el último tratamiento del cáncer renal, cuando afuera está quedando la cagá ( $\mathrm{sic}$ ) y yo cáncer renal no voy a ver en mi vida. Entonces tú quedas, como un poco que, todo el sistema intentó continuar como si nada estuviera pasando y eso igual es una sensación súper extraña, o sea, uno entra porque ya estas jodido, porque no puedo dejar de estudiar y ponerme a alegar con nadie, también no está mal la visión de querer que las cosas avancen, pero se siente súper extraño. (Enrique, Medicina US1, 2020)

\subsection{ADAPTACIONES PEDAGÓGICAS AL CONTEXTO ONLINE}

Sobre la modalidad de las actividades curriculares, los/as estudiantes afirman que las metodologías de trabajo en la mayoría de sus asignaturas siguieron siendo similares a las desarrolladas en las clases presenciales, es decir, eminentemente expositivas, refrendando lo señalado en la revisión de literatura sobre prácticas de coronateaching. Igualmente, de esto se desprende una crítica más amplia a la docencia universitaria que, en general, es concebida como poco innovadora y participativa, aspectos críticos que se incrementaron en las condiciones de educación remota. En este sentido, también se recalca que los/as docentes, al momento del cambio, estaban en una situación compleja y con pocos apoyos de parte de las instituciones de educación superior, más allá de algunas capacitaciones en softwares tecnológicos para el desarrollo de las clases online.

Las clases ahora básicamente han sido, el profesor hablando de un PPT, entonces yo igual de repente pienso, de que lo que es una clase presencial en sí, no es muy diferente pero lo que sí, es que de por sí disminuye la participación, el diálogo, la discusión, todo este tema, y yo creo que falta quizás, no sé, innovar quizás no solo en el tema online, sino que también en el tema presencial, cómo este esquema del profesor siempre frente de la pizarra hablando de un PPT lo que sea, siento que presencial y online, online es peor pero presencial también es malo, no es como que sea el dúo perfecto de que uno diga: «!Oh! Quiero ir a una clase presencial para ver al profesor hablando de un PPT». 
Al final yo siento que más allá de que sea online, o sea presencial, yo creo que es parte del esquema de los profesores en sí lo que está fallando, más allá de la modalidad. (Bastián, Bachillerato US2, 2020)

En el discurso predominante, los estudiantes valoran las clases y cursos prácticos y de aplicación de contenidos, que implican una participación más activa de su parte. Sin embargo, por el modo en el que están estructuradas las mallas de las carreras, los primeros años tienden a ser más bien de carácter teórico, lo que desalienta y cuestiona el ámbito vocacional de parte de los/as estudiantes. Junto con ello, existe una idea de poco trabajo y conocimiento pedagógico de parte de los docentes, quienes son expertos en sus áreas y los/as mejores del país, pero ello no se traduce en ser un/a buen/a profesor/a. Este juicio es transversal e independiente del tipo de carrera que estudian los entrevistados, haciendo visible un cuestionamiento a la gestión docente prepandemia que se agudiza en el marco de la emergencia y traslado de las actividades formativas a la modalidad online.

\subsection{CONDICIONES MATERIALES: APOYOS INSTITUCIONALES Y FAMILIARES}

Los/as estudiantes evidencian condiciones materiales y temporales disímiles, en consideración a su condición socioeconómica, espacio o territorio donde habitan, y género, tal como identifican las indagaciones revisadas (Cannellotto, 2020; IESALC UNESCO, 2020; Ordorika, 2020). Algunas problemáticas concretas que han enfrentado han sido el deterioro de equipos computacionales, por lo que deben renovarlos o compartirlo con alguien más del hogar; conexiones a internet débiles, que tiene relación con los lugares de residencia con baja conectividad; la falta de un espacio apropiado y de mobiliario necesario para su trabajo autónomo diario. Las instituciones de educación superior les han ofrecido apoyos y ayudas, con lo cual han recibido 
computadores, becas de internet, ayuda económica, entre otros. Sin embargo, algunos estudiantes señalan ciertas dificultades en estas materias, como ocurrió con la demora en acceder a equipos o sistemas de conexión necesarios para poder seguir la educación online, lo que los dejó, en un principio, en una situación de desventaja respecto a sus pares que sí tenían las condiciones necesarias. Por lo mismo, en al menos tres de las cuatro carreras de los estudiantes entrevistados, se verificó paralización de actividades exigiendo apoyo de las instituciones para estudiantes que no contasen con las condiciones requeridas.

Más allá de la disponibilidad de equipos y conexiones y las estrategias para su solución, un punto fundamental que afecta la organización de la vida universitaria en la modalidad a distancia de estos jóvenes, tiene relación con los lugares de residencia que, por lo general, son pequeños y con escasas posibilidades de aislamiento. Varios de los entrevistados señalaron compartir habitación con algún hermano; muchas de las casas habitacionales son pareadas y/o los lugares de residencia tienen altas concentraciones de ruido ambiente. Finalmente, llevar la vida de la universidad al interior de los hogares ha implicado irrumpir en el espacio de otros/as, usualmente padres y hermanos/as. Así, por ejemplo, en las entrevistas es recurrente el relato de las interrupciones por parte de familiares mientras están en clases o estudiando. Junto con ello, la vida en el hogar ha implicado la asunción de nuevas labores de cuidado, particularmente en el caso de la presencia de menores que también tuvieron que permanecer en el hogar debido a las medidas de restricción. Ante ello, los/as estudiantes afirman que entienden a sus familias y que ellos se sienten unos "intrusos" en su casa, y que su presencia irrumpe las rutinas familiares. En respuesta a esta situación, varios estudiantes han cambiado sus rutinas de estudio, muchos señalan dedicarse más horas a estudiar y realizar sus trabajos universitarios de noche, para poder hacerlos en un ambiente más propicio para el estudio.

Las situaciones más problemáticas las han experimentado los estudiantes que trabajan y estudian (incluyendo una entrevistada que, además, es madre); con 
todo, las experiencias son disímiles y evidencian lo complejo de sobrellevar un tiempo como el vivido. Roberto (Derecho US1) trabaja en el área que estudia y puede teletrabajar desde el hogar. Mientras que Javier (TS US1) trabaja part time, pero debe cumplir un horario que le ocupa gran parte del día, por lo que en la tarde noche y en la noche se dedica a sus actividades de estudio. Lorena (TS US1) trabaja en el día en distintas ocupaciones y cuida a su hijo, por lo cual también debe ver las clases de manera asincrónica durante la noche, además de leer y realizar los trabajos de los distintos ramos de su carrera. Si bien la mayoría de los entrevistados reconoce una disposición flexible de parte de los docentes y de las instituciones respecto a la formación en estas condiciones excepcionales, varios de estos observan que no se entiende o no se conoce a cabalidad su realidad. Tal como lo expresa uno de los estudiantes, muchos profesores aún tienen una noción de los estudiantes como pertenecientes a un cierto nivel social, que ha sido el perfil tradicional de estas carreras, ignorando la profundidad de las carencias y problemas cotidianos que deben hacer frente este grupo de jóvenes afectos a la gratuidad.

\subsection{EL FINANCIAMIENTO DE LA ES: SEGUIR COMO SEA}

Si bien las instituciones de educación superior han apoyado con una serie de iniciativas la continuidad de los/as estudiantes en este contexto de emergencia, igualmente aumentaron los aranceles de manera general en un plantel, y en una carrera en específico, en el otro. Dicha situación afectó a los estudiantes y su visión de las universidades, sobre todo considerando el contexto de crisis en el que nos encontramos, con familias que han disminuido o perdido sus ingresos (Ministerio de Desarrollo Social y Familia, 2020). Además, desde su perspectiva, las universidades estarían incurriendo en menores gastos bajo esta modalidad de enseñanza online, por lo que se hace menos comprensible esta alza. Con este marco de referencia es posible entender que, por primera vez en todo el proceso 
investigativo, surjan miradas clientelistas de parte de los/as estudiantes debido a estas alzas. En este punto es necesario tener presentes dos condiciones estructurales relevantes: primero, que las instituciones de educación superior dependen mayormente del pago de aranceles para su financiamiento y, segundo, que las universidades complejas, como las acá consideradas, incurren en gastos e inversiones que no solo incluyen la docencia, sino que investigación y extensión (Sanhueza y Orellana, 2018). De igual modo, los/as estudiantes resienten el trato de las instituciones en este contexto:

Yo siento mucha deuda con respecto a la universidad, la universidad dejó muy botados a todos sus estudiantes y siento que el costo de eso es súper grande, siento que el Covid va a generar una, no sé cómo decir, una herida, una cicatriz en todos los estudiantes que se están formando, que no va a poder reemplazarse, como repararse y que la universidad no dio lo que tenía que dar, para que eso fuera menos grave. (Enrique, Medicina US1, 2020)

Pucha, justo ahora cuando tengo $\mathrm{CAE}^{10}$ sube el arancel, y es más plata que tengo que pagar después, me da mucha rabia el tema, de que ni siquiera estamos yendo a clases, no estamos ocupando la infraestructura de la $\mathrm{U}$ y sigan con el mismo arancel. A mí me da rabia pagar, a lo mejor, algo que no estoy haciendo uso y aparte que no estoy aprendiendo como corresponde tal vez, así como no estoy obteniendo los aprendizajes que espero y al final voy a tener que pagarlo igual, entonces, igual me da mucha, mucha lata eso. Pero igual ya me hice la idea de que voy a pedir CAE, entonces ya no es un problema que me afecte, ya me hice la idea, ya me resigné. (Johanna, TS US2, 2020)

Aunque todos los estudiantes entrevistados han sido beneficiarios de la política de gratuidad en educación superior, algunos de ellos perdieron esta condición por excederse en el plazo formal de la carrera, por lo cual han debido solicitar créditos educativos (Fondo Solidario y Crédito con Aval del Estado). En este sentido, quienes mantienen la condición de beneficiarios de la gratuidad

\footnotetext{
${ }^{10}$ Crédito con Aval del Estado.
} 
manifiestan la sensación de alivio económico, más aún en este contexto de crisis financiera y de incertidumbre respecto al futuro. Por lo mismo, se mantiene y agudiza la preocupación por no atrasarse en la carrera ante la posibilidad de pérdida de este apoyo, mientras que quienes debieron recurrir al sistema de crédito se autoimpusieron la necesidad de tomar más carga académica en los siguientes semestres, y así acortar el tiempo extra que sea necesario realizar y financiar con crédito. De este modo, el propio mecanismo de gratuidad, que ha permitido a estos jóvenes ingresar y permanecer en sus carreras universitarias, ha operado en esta coyuntura como un factor de presión personal mayor al ya existente, que los obliga a hacer frente al desafío de cumplir con las exigencias curriculares independientemente de los problemas personales aquí reseñados. En efecto, ser beneficiarios de la gratuidad llevó a varios entrevistados a tomar la decisión de no congelar el semestre online, con el objetivo de no demorar el tiempo de titulación y egreso de sus carreras.

Hay que empezar a hacer la idea de que hay que pagar todo lo que salga de ahora en adelante, y además que con este tema del $\mathrm{CAE}$, del fondo solidario siempre se ven tantos casos de profesores que están sus 30, 40 años y siguen pagando, todo este tema ahora que está de la condonación del CAE, entonces uno dice, en qué me estoy metiendo ahora, qué va a pasar en el futuro, está todo este tema en la vida futura. (Bastián, Bachillerato US2, 2020)

Con el proceso de masificación, las instituciones de alta selectividad y prestigio se han visto enfrentadas a una mayor diversidad de estudiantes, en cuanto a sus orígenes sociales. No obstante, aún corresponden a un número menor en relación con el total de las carreras. De acuerdo con los datos entregados por Valenzuela (2021), las universidades de élite en Chile concentran en términos generales la matrícula en los dos quintiles más altos (entre 74 y 85\%), mientras que en carreras de élite dentro de estas mismas instituciones estos porcentajes aumentan (entre 83 y 91\%). Lo anterior puede explicar por qué los/as estudiantes beneficiarios de la gratuidad, aquellos que han accedido a becas y 
primera generación que estudia en estas instituciones tienen una sensación de abandono de parte de las instituciones, vinculada a la falta de comprensión de las situaciones complejas que algunos enfrentan en términos materiales, pues no corresponden al perfil histórico ni actual de estudiantes que acceden a dichas instituciones de manera mayoritaria y, por ello, pueden no ser del todo visibles y reconocibles.

\subsection{LA EXPERIENCIA SOCIAL: LA VIDA SOCIAL Y UNIVERSITARIA CANCELADA}

Las condiciones de prevención del Covid-19 han implicado la cancelación de actividades sociales. Aún así, se han propiciado encuentros online por medio de alguna plataforma de reunión. No obstante, en general, los/as estudiantes han tenido una baja vida social con sus pares de la universidad y ha sido un semestre más bien solitario. Tres estudiantes en particular señalan que con el paso del tiempo han ido retomando el contacto con sus compañeros/as por medio de reuniones virtuales, mientras que otros han realizado visitas presenciales a algunas amistades. A pesar de que muchos no tenían una vida social activa en el período previo a la pandemia, extrañan la posibilidad de ver a sus pares, tanto para estudiar como para conversar en los recesos entre clases y en las instancias e intersticios que posibilitaba la presencialidad.

Empezamos a hacer muchas más como reuniones sociales, nos hemos empezado a hablar más, eso ha fluido mucho más, porque antes también era un grupo bien ejecutivo y ahora ha sido más distendido, se ríe más, se habla más tonteras. Ha sido el cumpleaños de varios de ellos, por ejemplo, dos de ellos estuvieron de cumpleaños este mes, y ha sido súper bonito, porque hacemos instancias en que nos ponemos por cámara, estamos varias horas hablando y se ha sentido más como esta fraternidad un poco y en ese aspecto ha sido súper bonito, como reforzar eso, pero igual se demoró todo. Entonces tuvo su evolución todo. (Enrique, Medicina US1, 2020) 
Un resultado relevante de las entrevistas realizadas en la etapa anterior a la emergencia de la pandemia tenía relación con la apertura a la heterogeneidad que viven los estudiantes durante su experiencia universitaria (Lahire, 2004). En una sociedad tan estratificada y segregada como la chilena (PNUD, 2017), el paso por la universidad — y por estas universidades — había implicado el acercamiento a otros barrios, a otros pensamientos sociales y políticos, y a pares de otras realidades sociales de las que estaban acostumbrados en su experiencia escolar previa. Todo esto quedó en suspenso con la pandemia y así lo sienten también los/as estudiantes, quienes ahora viven la universidad desde sus hogares.

Que la universidad tiene muy, muy buena vida universitaria, que siempre hay fiestas o hay, no sé, algún panorama cultural, exposiciones, no sé, hay mucha actividad en la universidad, y todo eso se ha perdido completamente. Imagínate que ahora nuestra semana universitaria va a ser algo súper raro, no va a haber carrete, no va a haber fiesta, lo único que va a haber van a ser como unas presentaciones de unos humoristas a través de Zoom, entonces no sé, un cambio por lo menos en el ámbito de vida universitaria, del cielo a la tierra y nada, nada bueno, malo. (Guillermo, Derecho US2, 2020)

Que no estaba como el ambiente necesario al que estaba acostumbrado, por ejemplo, antes siempre estaba como una costumbre, que podía ir a la biblioteca, tomar cualquier libro, leerlo, consultar, cosa que aquí es difícil, no tengo nada para consultar, a dónde ir a pedir un libro. Y lo otro que después de clases, tenía la costumbre también de cualquier duda conversarla con el profesor, alguna inquietud, algún tema relacionado, lo mismo con los compañeros también, con los que más hablaba por aquel entonces y era básicamente ese acceso más directo a la información, de forma más presencial, más directa. (Bastián, Bachillerato US2, 2020).

Aún así, algunos estudiantes han encontrado espacios, vinculados a los estudios que cursan para colaborar en sus zonas residenciales. Así, Johanna (TS US2) ha sido parte de la organización de ollas comunes para enfrentar los problemas socioeconómicos en el barrio donde vive, mientras que Enrique (Medicina US1) colaboró con sus vecinos con conocimientos médicos sobre 
prevención del Covid-19, además de algunas prestaciones médicas menores. Estas actividades extraacadémicas como modo de colaboración con las comunidades en las que se asientan, les han permitido darles sentido a sus propios estudios, de manera extrauniversitaria, y reafirmar sus disposiciones vocacionales hacia las carreras en las que prosiguen estudios.

\section{A MANERA DE CONCLUSIONES}

Como se indicó, la experiencia de los/as estudiantes con el beneficio de la gratuidad en universidades altamente selectivas era compleja por sí misma en la etapa previa a la situación de reorganización de la cotidianeidad generada por la pandemia del Covid-19; las altas exigencias académicas y la necesidad de adaptación a un ambiente sociocultural más bien desconocido representan rasgos distintivos de este proceso y que dan cuenta, de manera patente, de la noción de desajuste del habitus en el marco del proceso de masificación de la educación superior. Sin embargo, como reportan las entrevistas realizadas en este estudio, la gran mayoría de los jóvenes, tras un proceso de adaptación que significó una alta agencia y disciplina, han logrado persistir en estas instituciones, cuestión asociada al ejercicio de un temple particular, una fuerte focalización en la vida académica, al apoyo de las mismas instituciones por medio de tutorías - claves para algunos estudiantes - y la disponibilidad de infraestructura y otros recursos necesarios para hacer factible el desempeño en condiciones de alta exigencia. Adicionalmente, la indagación reporta que los grupos de pares también resultaron ser fundamentales para sobrellevar la vida académica; aunque se trata mayoritariamente de vínculos de tipo instrumental y complejos, la percepción de contar con un soporte para responder a los desafíos de sus carreras constituía un capital en construcción para la gran mayoría de los entrevistados.

Sin embargo, este estado de situación se puso en suspenso con la pandemia y el traspaso de las actividades a la modalidad online. En efecto, esta 
experiencia académica a distancia ha significado el cierre de los lugares físicos de las universidades y, con ello, de los espacios de estudio y otros apoyos que resultaban claves para los/as estudiantes. Las instancias de estudio conjunto y los intersticios entre clases que permitían poner en discusión los contenidos vistos en las distintas disciplinas se suspendieron producto de las condiciones que exigen el enfrentamiento de esta pandemia, lo que ha obligado a los jóvenes a readecuar las rutinas de estudio generadas inicialmente.

En este nuevo escenario, la readecuación ha sido de carácter aún más individualizado, debiendo reorganizar rutinas desde el hogar en condiciones particularmente adversas, sin los apoyos institucionales que resultaban relevantes cuando podían concurrir a sus casas de estudio. En general, la experiencia a distancia propicia de manera transversal una relación instrumental con el conocimiento y una experiencia universitaria trunca, sin relación con los pares, con las instituciones ni con los sentidos de identidad profesional que propiciaba la vida universitaria prepandemia. De este modo, la percepción de los entrevistados es que se acrecientan brechas que en su momento pudieron ser reducidas por medio del uso de las instalaciones universitarias, y que sin embargo, en estas condiciones no resultan posibles. Lo anterior, por cierto, refrenda lo señalado por organismos internacionales respecto al aumento de las brechas educacionales en el contexto de pandemia (IESALC UNESCO, 2020; Pedró, 2020).

Como se indicó, un punto crítico lo constituyen las experiencias de práctica (tanto a lo largo del proceso formativo como en las etapas finales de las respectivas carreras), que en este contexto han sido de compleja adaptación para las instituciones. En esta transición entre el mundo universitario y laboral, los/as estudiantes resienten las carencias en la aplicación de los contenidos disciplinares que han aprendido durante años. Además, las experiencias vinculadas a la práctica han sido un punto clave en la construcción de un proyecto profesional 
en el marco de su experiencia universitaria. Dicho proyecto se pierde en este contexto (Dubet, 2010), aunque, como vimos en la sección anterior, algunos estudiantes han puesto al servicio de sus comunidades sus saberes profesionales, encontrando en estas acciones un sentido alternativo, con lo cual generan, de manera individual y por fuera de las instituciones universitarias, un proyecto profesional de incidencia social durante este tiempo.

Por cierto, muchos de los aspectos referidos a la situación de estos jóvenes no difieren mayormente a la experimentada por otros estudiantes que actualmente cursan estudios en la educación superior y que han debido adaptarse a una situación temporalmente extraordinaria. Sin embargo, es importante destacar la especificidad del caso de los jóvenes que motiva este trabajo en este nuevo escenario de crisis sanitaria, con el objetivo de discutir y revisar las políticas que enmarcan su condición de excepcionalidad.

En efecto, en los últimos años, el beneficio de la gratuidad y la inserción en carreras de alta exigencia de estudiantes que provienen de familias de escasos recursos han estado acompañados de acciones afirmativas desde los propios centros de estudios para apoyar una adecuada integración, buscando limitar las posibilidades de fracaso académico y/o deserción de sus carreras. De acuerdo a lo recogido en las entrevistas realizadas para este estudio, es justamente en este ámbito donde se observan las principales debilidades y desajustes institucionales para hacer frente a las nuevas condiciones de vida universitaria en situación de no presencialidad. La intermitencia o suspensión del acompañamiento y/o tutoría de estos estudiantes, sobre todo para estudiantes noveles, y la imposibilidad de suplir las condiciones materiales y de espacios físicos que proporciona la asistencia diaria a los centros de estudios, son dos de los factores más relevantes en esta dirección. Como contraste, la exacerbación de una lógica de resolución individual para el enfrentamiento de los desafíos académicos y la constatación cotidiana de que el espacio del hogar juega un rol adverso, a pesar del constante apoyo familiar en su proyecto educativo personal, representan las principales 
amenazas para el logro de las metas personales de estos jóvenes. Tales constataciones, creemos, deberían alimentar una agenda de discusión sobre las políticas de ampliación de oportunidades en la educación superior una vez superada la emergencia de la pandemia de Covid-19, buscando limitar así los principales factores de riesgo a las esperanzas y desafíos que estos jóvenes construyen en sus trayectorias en la educación superior.

RECIBIDO: 14 DE ABRIL DE 2021

RECIBIDO: 26 DE MAYO DE 2021

\section{BIBLIOGRAFÍA}

Abrahams, J. e Ingram, N. (2013). The Chameleon Habitus: Exploring Local Students' Negotiations of Multiple Fields. Sociological Research Online, 18(4), 213-226. https://doi.org/10.5153/sro.3189

ANDRÉU, J. (2002). Las técnicas de análisis de contenido: una revisión actualizada. Fundación Centro Estudios Andaluces, 10(2), 1-34.

Andrew, A., Cattan, S., D, Costa-Dias, M. C., Farquharson, C., Kraftman, L., Krutikova, S., Phimister, A. y Sevilla, A. (2020). Inequalities in children's experiences of home learning during the COVID19 lockdown in England. (Working paper, 20/26). The Institute for Fiscal Studies.

ARCHER, M. (2007). Making our Way through the World Human. Reflexivity and Social Mobility. Cambridge University Press.

ARISTOVNiK, A., KeRžǏč, D., RAVšELJ, D., TOMAŽEVIČ, N. y UMEK, L. (2020). Impacts of the COVID-19 Pandemic on Life of Higher Education Students: A Global Perspective. Sustainability, 12(20), 8438. https://doi.org/10.3390/su12208438

BerTAUX, D. (2005). Los relatos de vida: perspectiva etnosociológica. Bellaterra.

BRUNNER, J. J. (2009). Tipología y características de las universidades chilenas. Santiago de Chile: Centro de Políticas Comparadas en Educación, Universidad Diego Portales. 
CAnnellotto, A. (2020). Universidades viralizadas: la formación en y post pandemia. En I. Dussel, P. Ferrante y D. Pulfer (eds.), Pensar la Educación en tiempo de pandemia: entre la emergencia, el compromiso y la espera (pp. 213-230). UNIPE: Editorial Universitaria.

Centro de Estudios MINIDEC, CEM (2021). Niños, escuelas y COVID-19. Revisión de los principales artículos de investigación. Evidencias $\mathrm{n}^{\circ} 51$. Recuperado de: https://centroestudios.mineduc.cl/wpcontent/uploads/sites/100/2021/03/EVIDENCIAS-51_2021.pdf

Di Pietro, G., Biagi, F., Dinis Mota Da Costa, P., Karpinski, Z. y Mazza, J. (2020). The likely impact of COVID-19 on education: Reflections based on the existing literature and recent international datasets, EUR 30275 EN. Publications Office of the European Union, Luxembourg. doi:10.2760/126686, JRC121071. Recuperado de: https://publications.jrc.ec.europa.eu/repository/handle/JRC121071

Dubet, F. (2010). Sociología de la Experiencia. Madrid: Centro de Investigaciones Sociológicas (CIS).

Dussel, I., Ferrante, P. y Pulfer, D. (2020). Presentación. En I. Dussel, P. Ferrante y D. Pulfer (eds.), Pensar la Educación en tiempo de pandemia: entre la emergencia, el compromiso y la espera. UNIPE: Editorial Universitaria.

ESPINOZA, V. y BAROZET, E. (2008). ¿De qué hablamos cuando decimos «clase media»? Perspectivas sobre el caso chileno. Expansiva-UDP.

Gallardo, G., Lorca, A., Morrás, D. y Vergara, M. (2014). Experiencia de transición de la secundaria a la universidad de estudiantes admitidos en una universidad tradicional chilena $(\mathrm{CRUCH})$ vía admisión especial de carácter inclusivo. Pensamiento Educativo: Revista de Investigación Educacional Latinoamericana, $51(2)$, 135-151. https://doi.org/10.7764/PEL.51.2.2014.10

Guzmán-Valenzuela, C., Rojas-Murphy Tagle, A. y Gómez-González, C. (2020). Polifonía epistémica de la investigación sobre las experiencias estudiantiles: el caso Latinoamericano. Education Policy Analysis Archives, 28, 1-36. https://doi.org/10.14507/epaa.28.4919

Hussein, E., Daoud, S., Alrabaiah, H. y Badawi, R. (2020). Exploring undergraduate students' attitudes towards emergency online learning during COVID-19: A case from the UAE. Children and Youth Services Review, 119, 105699. https://doi.org/10.1016/j.childyouth.2020.105699

IESALC UNESCO (2020). COVID-19 y educación superior: De los efectos inmediatos al día después. Recuperado de: 
http://www.iesalc.unesco.org/wp-content/uploads/2020/05/COVID-19-ES130520.pdf

KREMERMAN, M. y PÁEZ, A. (2016). Endeudar para gobernar y mercantilizar: El caso del CAE. Fundación SOL.

LAHIRE, B. (2008). The individual and the mixing of genres: Cultural dissonance and self-distinction. $\quad$ Poetics, 36(2-3), 166-188. https://doi.org/10.1016/j.poetic.2008.02.001 . (2004). El hombre plural. Los resortes de la acción. Barcelona: Bellaterra.

LEHMANN, W. (2009). Becoming Middle Class: How Working-class University Students Draw and Transgress Moral Class Boundaries. British Journal of Sociology of Education, 30(2), 137-149. https://doi.org/10.1177/0038038509105412

LeY EdUCACIÓN SuPERIOR (2018). Biblioteca del Congreso Nacional. Recuperado de: http://bcn.cl/2fcks

Leyton, D., VÁsquez, A. y Fuenzalida, V. (2012). La experiencia de estudiantes de contextos vulnerables en diferentes Intituciones de Educación Superior Universitaria (IESU): Resultados de investigación. Calidad en la Educación, 37, 61-97. https://doi.org/10.4067/S071845652012000200003

MCNAY, L. (2004). Agency and Experience: Gender as a Lived Relation. The Sociological Review, 52(2_suppl), 175-190. https://doi.org/10.1111/j.1467954X.2005.00530.x

. (1999). Gender, Habitus and the Field. Theory, Culture \& Society, 16(1), 95-117. https://doi.org/10.1177/026327699016001007

Merrill, B. y West, L. (2009). Using Biographical Methods in Social Research. https://doi.org/10.4135/9780857028990

Ministerio De Desarrollo Social y FAmilia (2020). Encuesta Social COVID-19.

Mishra, L., GuPTA, T. y ShreE, A. (2020). Online teaching-learning in higher education during lockdown period of COVID-19 pandemic. International Journal of Educational Research Open, 1, 100012. https://doi.org/10.1016/j.ijedro.2020.100012

OECD (2020). Youth and COVID-19. Response, recovery and resilience. https://read.oecd-ilibrary.org/view/?ref=134_134356- 
ud5kox3g26\&title=Youth-and-COVID-19-Response-Recovery-andResilience

ORDORIKA, I. (2020). Pandemia y educación superior. Revista de la educación superior, 49, 1-8.

Orellana, V. (2011). Nuevos estudiantes y tendencias emergentes en la educación superior una mirada al Chile del mañana. En M. JiMÉNEZ y F. LAGOS (eds.), Nueva geografía de la educación superior y de los estudiantes: Una cartografía del sistema chileno, su actual alumnado y sus principales tendencias (pp. 80-140). Santiago de Chile: Ediciones Universidad San Sebastián.

Pedró, F. (2020). Covid-19 y educación superior: crisis y ventanas de oportunidad. En I. Dussel, P. Ferrante y D. Pulfer (eds.), Pensar la educación en tiempo de pandemia II. Experiencias y problemáticas en Iberoamérica (pp. 73-85). UNIPE: Editorial Universitaria.

Pensiero, N., Kelly, A. y BoKhove, C. (2020). Learning inequalities during the Covid-19 pandemic: how families cope with home-schooling. University of Southampton research report. doi: 10.5258/SOTON/P0025

PÉrez CEnTENO, C. (2020). La educación superior en América Latina. Situación y futuros frente a la emergencia del covid-19. En I. Dussel, P. FERRANTE y D. Pulfer (eds.), Pensar la educación en tiempo de pandemia II. Experiencias y problemáticas en Iberoamérica (pp. 87-97). UNIPE: Editorial Universitaria.

PÉreZ-RoA, L. (2014). El peso real de la deuda de estudios. La problemática de los jóvenes deudores del sistema de financiamiento universitario de la Corfo pregrado en Santiago de Chile. Education Policy Analysis Archives. https://doi.org/10.14507/epaa.v22n75.2014

PNUD (2017). Desiguales. Orígenes, cambios y desafíos de la brecha social en Chile. Programa de las Naciones Unidas para el Desarrollo.

REAY, D. (2010). From the theory of practice to the practice of theory Working with Bourdieu in research in higher education choice. En E. Silva y A. WARDE (eds.), Cultural Analysis and Bourdieu's Legacy (pp. 75-86). Routledge. https://doi.org/10.4324/9780203878620

ReAy, D., Crozier, G. y Clayton, J. (2010). «Fitting in» or «standing out»: working-class students in UK higher education. BERJ, 36(1), 107-124. https://doi.org/10.1080/01411920902878925

. (2009). «Strangers in Paradise»? Sociology, 43(6), 1103-1121. https://doi.org/10.1177/0038038509345700 
ROJAS, M. (2008). Lo biográfico en sociología. Entre la diversidad de contenidos y la necesidad de especificar conceptos. En M. L. TARRES (ed.), Observar, escuchar y comprender. Sobre la tradición cualitativa en la investigación social (pp. 171-197). FLACSO.

SAnhueza, J. M. y Orellana, V. (2018). Análisis crítico de la reforma educacional de Bachelet (2014-2018). En Entre el mercado gratuito y la educación pública. Dilemas de la educación chilena actual (pp. 209-257). Santiago de Chile: LOM.

SAUTU, R. (1999). El método biográfico. La reconstrucción de la sociedad a partir del testimonio de los actores. Buenos Aires: Editoral de Belgrano.

Scott, J. W. (2009). Género e Historia. México, D. F.: Fondo de Cultura Economica.

Stillerman, J. (2016). Educar a niñas y niños de clase media en Santiago: capital cultural y segregación socioterritorial en la formación de mercados locales de educación. Revista EURE, 42(126), 169-186.

Toledo, M. I. (2002). Enfoque biográfico: marco teórico-metodológico para la construcción de relatos de vida. Parte II. Revista Praxis, 4, 39-48.

TORCHE, F. y WORMALD, G. (2004). Estratificación y movilidad social en Chile: entre la adscripción y el logro. Naciones Unidas, CEPAL, División de Desarrollo Social. https://repositorio.cepal.org/handle/11362/6089\#.XyfUCEWjVN4.mendel ey

TORRES, R. y ZENTENO, M. (2011). El sistema de educación superior. Una mirada a las instituciones y sus características. En M. JIMÉNEZ y F. LAGOS (eds.), Nueva geografía de la educación superior y de los estudiantes: Una cartografia del sistema chileno, su actual alumnado y sus principales tendencias (pp. 80-140). Santiago de Chile: Ediciones Universidad San Sebastián.

Valenzuela, J. P. (2021). Universidades de élite en Chile: Composición, tendencias y cambios en la última década. «Seminario Educación y Élites en Chile. Debates, Investigaciones y Aportes». Enlace: https://www.youtube.com/watch?v=QimlffMzNvY

Villalobos, C., Quaresma, M. L. y Franetovic, G. (2020). Mapeando a la élite en las universidades chilenas. Un análisis cuantitativomultidimensional. Revista Española de Sociología, 29(3), 523-541. https://doi.org/10.22325/fes/res.2020.33 